\title{
The Historical Aspect of the Development of Quantum Consciousness at the Beginning of the XXI Century
}

\author{
Adam Adamski* \\ Faculty of Ethnology and Educational Science in Cieszyn, Poland \\ *Corresponding author: Adam Adamski, Faculty of Ethnology and Educational Science in Cieszyn, Poland
}

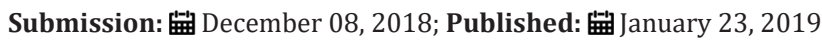

\begin{abstract}
The biochemical model explains the complex mechanisms of mental life seems to be correct. The transmission from inanimate matter to living matter cannot be explained. He still cannot explain the nature of consciousness and the transition from inanimate matter to living one. Where is the threshold and what is the role of biochemical processes in consistency of soma and consciousness as well as in building a mental structure. The author supports the thesis that the nature of mental processes is inexplicable as far as interactions of biochemical processes are concerned and it is much easier to describe it in the light of quantum processes.
\end{abstract}

Keywords: Coherent light; Bioplasma consciousness; Soliton

\section{Introduction}

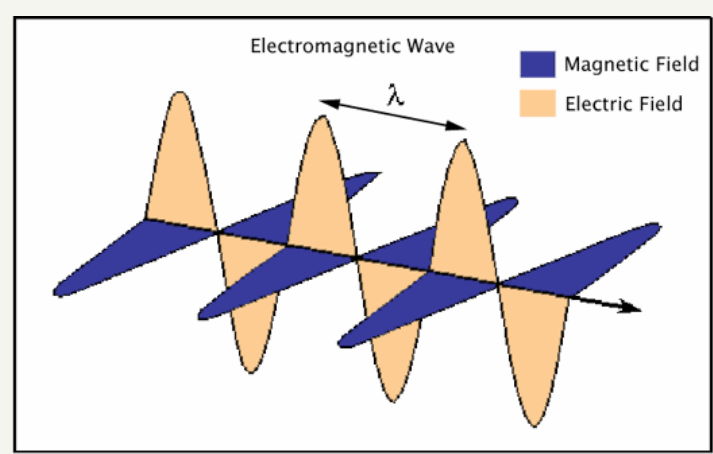

Figure 1: Shows the electromagnetic wave.

Quantum psychology is able to accept the thesis that consciousness in its process of operation may behave as a wave or a particle, and it can also include linear and nonlinear processes. The existing biochemical model fails to account for the ultimate comprehension of the nature of life, consciousness, and other mental functions. Besides the traditional, well-known chemical reactions, a new reality based on the bioelectronic model of life now appears. This model assumes that the biological system is the carrier of the electronic data in which the cell fulfils the role of the Nano computer [1]. The microtubules and cytoskeleton are considered as microprocessors, and enzymes are regarded to be transistors [2]. The proteins, DNA, melanins and RNA are an electronic material of piezoelectric, pyroelectric, and semi conducting properties, and have an ability to encode information which can then be deciphered by the brain Figure 1. One is physically and mentally bonded with the whole surrounding world. One's body is a center of electromagnetic phenomena, gravitational forces, and all kinds of chemical reactions and bioelectronic processes responsible for the development of mental and biological functions [3-6]. The authors show a close functional correlation on the role of the electronic process shaping human perception and mental functions, including the all biological system which is a function the biochemical and bioelectronic processes. These processes show a close functional correlation. A significant factor linking chemical and bioelectronic processes is called bioplasm [7].

\section{Soliton concept of consciousness [8]}

Tubular cytoskeleton system consisting of hollow cylinders, which walls are equipped with a hexagonal molecule tubulin functions in all brain cell tissues. The ends of the tubules are filled with concession. This system creates a tubular connectivity for the entire cell and brain. In living cells, not only nucleic acids and proteins but also water and enzymes have the ability to process and transmit information. Water due to the dipole structure is an important aspect of quantum. Changes of the angle of aperture create a rotational motion component; dipole water starts to spin to the right or left, creating a pair of spin. In aqueous tubular cytoskeleton soliton waves are formed, which flow without distortion in a different direction, bringing information to the cell. Protein tubulins are surrounded by water dipoles. Solvation coat may be of different thickness, which determines the rise of gel physical state. 
Gel coat inhibits the movement of tubulin and soliton in tubular cytoskeleton. Such a phenomenon is observable at the time of anesthesia and results in changes of consciousness, it means that the solitons, along with the eddy fields are responsible for states of consciousness. Solitons due to tunneling and superconductivity phenomenon spread rapidly and the whole organ does not respect the boundaries of cells. Solitons require the presence of the physical environment as a base medium, and therefore they cannot propagate in a vacuum, which do not require other forms of energy Figure 2. Solitons do not transfer molecules, but only information contained in the change of conformation, or transformation of the environment of water, gas etc. Such interactions form the system of mutual communication in brain and may also pass in the space outside the brain. Interaction solitons is resistant to deformation which facilitates extra cranial communication [8].

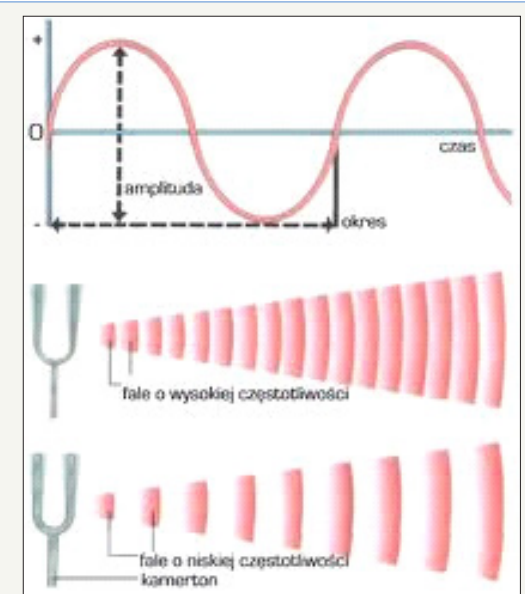

Figure 2: Shows the acoustic wave.

\section{Soliton concept of consciousness [9]}

The nonlinear mechanism for the origin of the weak biophoton emission from biological systems is suggested. The mechanism is based on the properties of solitons that provide energy transfer and charge transport in metabolic processes. Such soliton states are formed in alpha-helical proteins. Account of the electronphonon interaction in macromolecules results in the selftrapping of electrons in a localized soliton-like state, known as Davydov's solitons. The important role of the helical symmetry of macromolecules is elucidated for the formation, stability and dynamical properties of solitons. It is shown that the soliton with the lowest energy has an inner structure with the many-hump envelope. The total probability of the excitation in the helix is characterized by inters pine oscillations with the frequency of oscillations, proportional to the soliton velocity. The radiative lifetime of a soliton is calculated and shown to exceed the life-time of an excitation on an isolated peptide group by several orders of magnitude. The conception is connected with the hypothesis.

\section{Electromagnetic theory field consciousness called the theory “CEMI" [10]}

The human body consists of about one hundred billion neurons electrically active producing electro-magnetic field which role in neuronal processing has not been fully investigated. In his book titled "Quantum evolution," McFaden presents evidence that the source of quantum effects in the brain is not associated with microtubules, but with the interaction between electromagnetic fields and voltage of the brain ion channel in the neuron membrane. Ion channels are very sensitive to electrical and electromagnetic field and are older than the structure of microtubules. The brain generates an electromagnetic field that affects the functioning of neurons. Information is collected in neurons (merged, integrated) in one unit and is reflected back into the neurons through the brain's magnetic field [10]. Consciousness and attention of humans and animals is the result of field 'em" in the brain, caused by the synchronization of nerve impulses Figure 3.

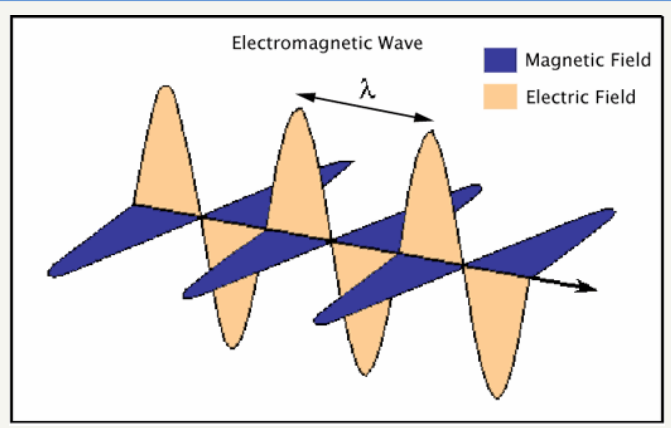

Figure 3: Shows the electromagnetic wave.

Factor, which causes disruption in the interaction between electromagnetic field of neurons brain will induce unconsciousness. Arousal and alertness will interact with the fluctuating field "em" brain. The brain should be protected from exposure to electromagnetic fields, since this leads to psychobiologic disturbances [10]. The conscious electromagnetic field theory (CEMI field theory) described above proposes that consciousness is a manifestation of the brain's electromagnetic (EM) field. The key feature of the brain's EM field is that it is capable of integrating vast quantities of information into a single physical system and it thereby accounts for the binding of consciousness. The CEMI field theory is shown to be compatible with all the known facts about con-sciousness. Unlike quantum theories of consciousness, the CEMI field theory does not require any special physical states in the brains; it is perfectly compatible with brain physiology. Nevertheless, recent work has shown that classical electromagnetic waves may be used to implement quantum algorithms; therefore, the brain's CEMI field may be able to perform quantum computations (but without the requirement for quantum coherent states of matter).

\section{Spin mediated consciousness [11]}

Hupping $\mathrm{Hu}$ and Maoxin Wu postulate that consciousness is intrinsically connected with quantum mechanical spin since spin is embedded in the microscopic structure of space-time and may be more fundamental than space-time itself. Thus, we theorize that consciousness emerges quantum mechanically from the collective dynamics of "protopsychic" spins under the influence of space-time dynamics. That is, spin is the "pixel" of mind. The unity of mind is achieved by quantum entanglement of the mind-pixels. Applying these ideas to the particular structures and dynamics of the brain, we postulate that the human mind works as follows: The team of 
nuclear spins "(NSE)" in both neural membranes and proteins form the basis for creating quantum processes of consciousness.

Information is communicated to NSE through strong spinspin couplings by biologically available unpaired electronic spins. Diffusion of oxygen molecules and nitrogen oxides is strongly associated with spin state processes which are information for the brain. On the other hand, the dynamics of NSE has effects through spin chemistry on the classical neural activities such as possibility of action and receptor function influencing in this way the classical neural networks of the brain. Our proposal calls for associative encoding of neural memories in the dynamical structures of neural membranes and proteins. Therefore, according to the present theory: (1) the dynamical nuclear spin ensembles are the "screen" of mind with nuclear spins as its pixels; (2) the neural membranes and proteins are the mind-screen and neural memory hosts; and (3) the fluxes of biologically available unpaired electrons spins are the spin beam for information retrieval, communication and pixel-activation. Together, they form the neural substrates of consciousness. We also present supporting evidence and make important predictions. Hupping $\mathrm{Hu}$ and Maoxin $\mathrm{Wu}$ underline that their theory is experimentally verifiable with present technologies. Further, experimental realizations of intra-inter-molecular nuclear spin coherence and entanglement, macroscopic entanglement of spin ensembles and NMR quantum computation, all in room temperatures, strongly suggest the possibility of a spin mediated mind Figure 4.

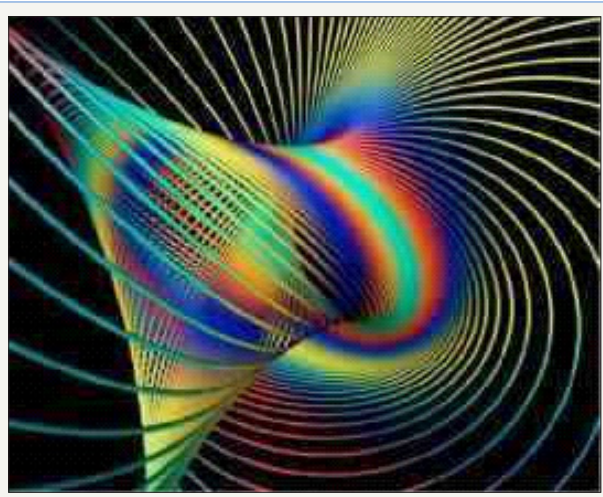

Figure 4: Left and right torsion fields.

\section{Quantum theory and the foundations of perception [12]}

[12] suggests that the ultimate level of description of mind and brain ought to be the quantum level, insofar as quantum field processes mediate all chemical and biological processes.

Flanagan explores a number of possibilities:

A. Whether quantum fields constitute the ultimate level of description of biological systems;

B. Whether this ultimate level is nested within higher levels of organization by way of the kinds of self-similarity found in neural structures;

C. Whether this lowest level embodies the initial conditions upon which the brain exhibits sensitive dependence.
The thesis is that perceptual fields are coordinated with photon fields. Therefore, the irreducibility of the secondary qualities flows from their elemental character and this might be in the form of:

A. Internal state spaces of gauge theory;

B. Additional spatial dimensions of string/M-theory;

C. Hidden variables theory.

Flanagan argues that color vectors, considered as -immediately experienced qualities together with their configurations in the visual field, ought to map to photonic vectors and their configurations in a photon field: vectors would get mapped to vectors, and fields to fields.

\section{The second order brain and the conscious mind [13]}

One way to avoid the Copenhagen/von Neumann metaphysics, while retaining a physical sense for the "collapse", is the concept of "decoherence". At first sight any isolated (relatively) small microscopic system would be "coherent", in the sense that entanglement of particles/waves would be the rule, and any (relatively) large macroscopic system would be "decoherent", in the sense that any property of this system, available to macroscopic observation and experimentation, would reveal no signal of entanglement between its elements. A widespread belief that explains why nature behaves in this way is called "law of large numbers", that identifies the size of the system as the critical parameter for statistical cancellations to apply, thus generating a most probable behavior that would correspond almost exactly to the predictions made by classical physics. In this view, the existence of quantum coherence at the macroscopic level would be extremely improbable and could not frequently and continuously occur in the conscious brain.

For the defenders of the quantum mind hypothesis, or even for physicists who have worked with quantum macroscopic effects, there must be something wrong with such usage of the "law of large numbers". Therefore, if the "law of large numbers" is not universal and/or is not really a physical law, a theoretical possibility is open, for a large macroscopic system as the brain, to be continuously generating quantum coherent processes among particles/waves distributed along its volume. These processes would function as a "second-order brain", controlling and being controlled by classical processes that occur in that same system (the "first-order brain"). This "second-order brain" would be the ultimate support for the flux of experiences called "the conscious mind" [14].

\section{Biophoton theory of consciousness [15]}

Fritz Albert Popp has shown that the photons are involved in the transmission of information within a cell and between cells. He showed that DNA of living cells stores and releases photons. He called this "biophoton emission". The intensity is about 1018 times lower than regular daylight. To study this phenomenon, he constructed very sensitive instrument which could detect the glow of a firefly 10 miles away. DNA uses a variety of frequencies as an information tool suggesting a feedback system of perfect communication 
through waves which encode and transfer information. Another fascinating characteristic of photons is their coherence. In a healthy state the emission is more coherent than anything that one has ever developed. Quantum coherence means that subatomic particles are able to cooperate. These waves know about each other and are highly interlinked by bands of electromagnetic fields. They can communicate with each other. It is analogous to an orchestra where all photons are playing together but as individual instruments that are able to carry on playing individual parts Figure 5. Therefore, biphotonic emission is a perfect communication system that transfers information to many cells across the body and to other bodies. According to Popp, biophotons are responsible for awareness, because light is the governing factor that determines the processes of our consciousness.

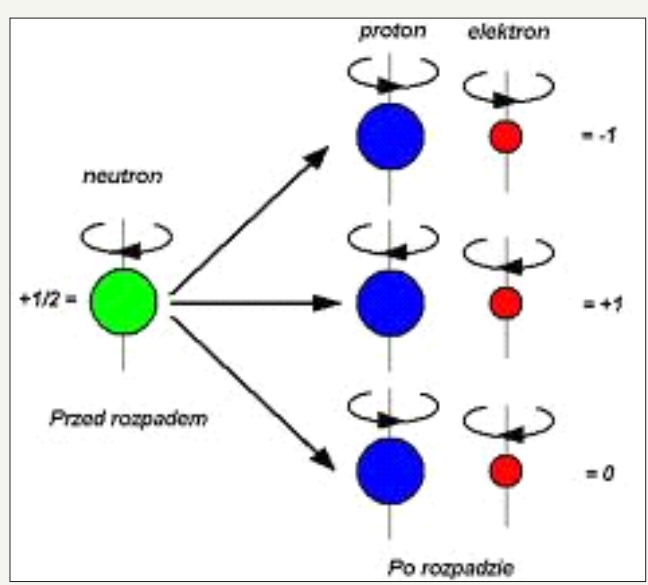

Figure 5: Shows the movement of spin, - to the right or left.

The organism-environment theory: consciousness and the ultimate essence of matter [16]

Argues that the understanding of the ultimate essence of matter depends essentially on the conception of human consciousness [16]. In the framework of the organism-environment theory, consciousness that develops with human cooperation and communication is the basis of any description of the world. Järvilehto claims that the properties of matter are relations created in human action/measuring. Therefore, there is no ultimate essence of matter, and the question "What exists?" is identical with the question -What can we know? According to the organismenvironment theory the world that may be described appears with the appearance of human consciousness. Consciousness was created in a system of several individuals when their actions were joined in the achievement of common results.

This joining was possible through communication which later developed into language. As communication was needed primarily for the production of common results, language developed primarily for the description of the common results that were intended or achieved. This means that words were not, in the first hand, used for the description of the world, but they rather acted as indicators of common results. Therefore, language (and also personal consciousness mediated by the language) was primarily directed towards generation of the cooperative organization. Thus, a word is basically not a symbol representing something, but a proposal for common action. It is precisely the common result that is stored in language, and, therefore, the language reflects the history and culture of humankind. Language is the historical collection of the results of human cooperation. Those parts of the universe which became objects of language were primarily those that were needed by human beings in their actions in relation to their bodies. Therefore, the perceived (conscious) structure of the world reflects more the structure of the human body, as it may fit the environment, than any independent structure of the world. The implicate order of the universe for an individual is the implicate order of the human body in its environment Figure 6.

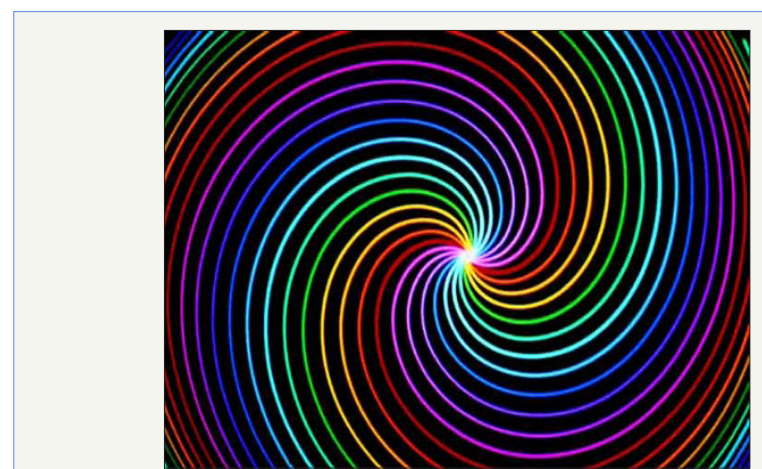

Figure 6: Shows the formation of solitons.

\section{Belal Baaqie and François Martin 2005-Quantum field theory of the human psyche}

Baaqie and Martin consider the human psyche to be a part of a universal phenomenon, and not only specific and unique to human beings. Human psyche is described as a superposed state. In quantum mechanics, the spin of an electron has two forms of Existence, namely the physical and the virtual. During observations, it is in a physical state in the spin which either points up or down. On the other hand, if it is not being observed, the spin is in a virtual state in which the spin can simultaneously exist in two mutually exclusive states. Every time the spin is observed, it is found to point in one direction only. Hence the virtual state can never be directly observed. nevertheless, only the human mind can see the virtual state. It follows the human psyche that itself must possess a similar virtual state, which is required to recognize its surrounding reality.

\section{Bioplasm concept of consciousness [17]}

In our discussions on consciousness, we encounter many difficulties, which arise from the lack of unified theory of consciousness, both in the natural sciences and psychology. Do we really know, what consciousness is in the ontological sense? Whether it is the structure, process, act, product, or any other phenomenon? Human consciousness includes not only knowledge of reality, but also self-knowledge or awareness of the human mind itself by its own actions, feelings, thoughts and motives of conduct, its own situation in the world and society. Awareness of oneself is a main characteristic of personality but it is not an innate characteristic. It is developed gradually in the course of our lives from early childhood through school years, adolescence, to adulthood [18]. 
In psychology, according to Z. Zaborowski, consciousness is understood as a process of encoding, processing information about oneself and integrating with the surrounding world. In psychology, encoding process is seen as attention, information processing relates to memory and thinking, and integration of information is associated with consciousness. Reflective self-consciousness is associated with integrating information about oneself on the basis of notions, patterns and abstract thinking. It is the highest stage of human psychological development, it has a factor integrating mental phenomena such as memory, attention, thinking, imagination, emotional experiences that have developed in the course of human evolution [19]. Self- consciousness gives us a sense of personal history and identity. It conforms functions to integrate, select and reconcile information related to one's own person. Not only does it cover the actual experience of individuals, but it is also able to return to the past appreciation of the course of one's life, one's difficulties, mistakes, achievements, and plan the future tasks and goals in life [19]. Consciousness co-works with the brain, but the brain can function at different stands: sleep, anesthesia, meditation, being under the influence of drugs.

A disease and above-mentioned states cause impairment of consciousness, called altered states of consciousness. In these altered states of consciousness distorted observations, such as visual perception distortion of time and space, the sense of separation from the body's own self, needs and problems of life appear. There may also occur glare, joy, ecstasy and a sense of fusion with others and the world. Awareness may have also nature of act of cognition, of decision, of imagining something. W. Sedlak recognizes that the integrating factor of the biological system is bioplasm. Penrose, Hameroff, Frolich, Zohar believe that the biosystem has its consistency due to Bose-Einstein condensate. According to FA Popp coherent light emitted from DNA is used to create a Bose - Einstein condensate and the structure of consciousness. Light is also used for intercellular communication. Biophoton radiation is seen as light with a high degree of coherence. This consistent information is able to repair the faulty system and lead to a harmonious state.

Popp considers that it can manage the process of consciousness and it is responsible for its nature, because light is determining element of the life process. In order to answer the question about the nature of bioplasm in W. Sedlak's terms, it would be necessary to start specific research. Plasma is a natural center, which combines chemical and physical process. Changes in chemical composition and physical factors affect changes in plasma's concentration, particle mass, the size of its electric and magnetic charge, which lead to changes of oscillation. Plasma has a great natural dynamicsall of its particles constantly "feel" the behavior of other particles, responding to the changes in their movement. Movement of each particle endowed with a cargo is made with a number of other oppositely charged particles. This property is a consequence of the impact of electric and magnetic fields and changes in dielectric properties of the medium. Plasma has many properties that are not observable in other physical states.

One of its most important features is a collaborative community response to all particles on the distortion of the balance. Interaction of particles in the plasma leads not only to direction change but also to the speed exchange of energy between the particles. As a result of collisions, a particle undergoes a small angular displacement. This means that there is a change of momentum of the particle, and therefore in the process of collision, defined part of the kinetic energy of accelerated particles will be transferred to the partner. Plasma responds to magnetic and electric fields. An important attribute of bioplasm is combination of different kinds of fields and particles [20]. Therefore, it is concluded that bioplasm is characterized by a common system for wave energy and mass (particles such as electrons, photons, phonons [21].

Bioplasm is organized by its surroundings, but also has the ability to self-organization, which may occur by the magnetic fields generated by the orderly movement of a sufficient number of charged particles. It is a phenomenon known as plasma pinch. Plasma is a natural material and the material life of the universe [22]. Bioplasm is linked to life's processes (metabolism and electronic processes in semiconductors protein [23]. According to Sedlak, bioplasm has its own " diffraction grid", as well as electromagnetic and acoustic grid. Therefore, any disturbance of the power grid is information for the biosystem. Bioplasm "knows" what happens in and around. Protein semiconductors, piezoelectric or organic compounds such as melanin, neuromelanin, melatonin, DNA, RNA, etc. habit bioplasm. In order to function properly, it needs different forms of energy. Plasma does not last, it is created and lost. In this process outside energy plays an important role. Receiving information from the outside forces the body to switch appropriately on its own power. Divert energy that could achieve the specified information for biosystem must go through the transformation of a uniting factor within the system. Otherwise there could occur a chaotic accumulation of random changes in the environment. Plasma is just such a state of matter, which is unified in its diversity and carries the mark of an integrating factor information. Changing the information disclosed in the electrical profile changes plasma biofield. This refers to changes in temperature, pressure, gravity, electric and magnetic fields, torsion, chemical, acoustic and optical changes. Each type of energy delivered to the plasma increases the electrical symmetries, gives the speed of particles and prevents destabilization processes, namely degradation of bioplasm [21].

In the biological system of human being, consciousness and bioplasm should have ascribed the same properties. At the bottom of the quantum of life, there is no difference between life and consciousness, between the psyche and the BIOS. There is unity and the quantum level in the protein environment forms a vital link between the electrons, photons and phonons in piezoelectric medium organic semiconductors. Here interaction between the chemical and electronic process merges [24]. As an example of the above-mentioned observations serves ontogenetic development of an individual. In this development, pre-school and adolescence periods play an important role. The period of adolescence is a period of enormous changes in biological, physiological, but also in psychological system. These changes activate the processes of quantum states that have an impact on the development of bioplasm in the brain and the whole biological system. 
Increased level of bioplasm in the nervous system of an individual contributes to the emergence of reflective consciousness [23]. The final stage phase of ontogeny may be characterized by weakening of the degree of collectivity in interactions within the clusters in the plasma biosystem, decrease in the coupling between them, or by the disintegration of the plasma in plasma's clusters. The disintegration of the plasma from the organ can lead to dysfunction of the entire biological system and ultimately to its death. A similar point of view about bioplasm presents Inyushin. According to him, physical fields that are present in bioplasm, should create a biological field. Bioplasm takes various condensation values in different parts of the organism, because the particles creating bioplasm can move around the whole biological system.

However, bioplasm task is to keep balance of carriers condensation in the specified proportions for particular parts of the system. The biggest concentration of electron- holes and electronproton bioplasm as well as satiation of wave processes are visible in the brain, spinal cord, peripheral nerves and receptor cells. The brain, however, has the highest level of structuralized somatic bioplasm. Proton-electron bioplasm is characteristic for cell nuclei [25-27]. In the theories presented by different authors, one sees that the key words are as follows: the electromagnetic spin, torsion fields, or Bose-Einstein condensate. Significant role in the formation of mental and sensory states plays melanin, neuromelanin and melatonin. These substances in the human ontogenesis play a very important role in shaping adolescence and reflective consciousness, abstract thought and higher feelings.

The period of adolescence is characterized by a continuous increase of melanin and melatonin in the child's biological system. After that period, quantity of these substances is reduced. This fact effects the growth of free radicals in a child's biological system which influence the development of mental processes. This results in increased activity of different torsion fields (vortex fields) that have an impact on the growth of a density wave bioplasm and this in turn gives rise to the development of the mind in the form of abstract thinking, and reflective consciousness. Melanin has the ability to absorb reactive hydroxyl free radicals, quenching singlet oxygen and excited states of some photo sensitizers, and inhibition of lipid peroxidation [28], on the other part to produce radicals. In this context, melanin can act as both an oxidant and as a reducer [29].

The most important feature of melanin is its ability to absorb light and retention, to store and recover energy. It protects against oxidative stress by reactive free radicals: peroxyls, hydroxyls and singlet oxygen quenching of excited states [30]. Melanin is made up of neurotransmitters and is able to change a light in tone, and vice versa. Color and light are processed in the music for the whole body. Each frequency produces its own color note [31].

In terms of electronics and physics, melanin is characterized by the following features:
A. Donor-acceptor properties;
B. The ability to proton conductivity Matuszak 2001

C. The ability to absorb all wavelengths of light;

D. Properties of photoconductor and amorphous semiconductor [32],

E. Increased resistance to light and ultraviolet light;

F. Generation of electrons and photons

G. High demand for oxygen [33].

H. Selective vulnerability to phonons, which means that cells with melanin are selectively sensitive to sound waves. Sarna, Swartz 1994.

I. Melanin may function as a transmitter of photons and phonons in the process of reverse [5]

J. Paramagnetic properties [34].

K. Melanin is piezoelectric and pyroelectric, and that fact allows it to transform mechanical, thermal acoustic energy, electricity, and with it the electric field.

L. Aggregate all types of metal ions [35].

\section{Melanin converts the light into sound}

Melanin converts the electromagnetic wave in the acoustic wave, photon in phonon and vice versa: the phonon in the photon [5]. Corry, Mc. Gines, Armour 1976. Melanin has the ability to direct light, it can accelerate or delay its movement.

\section{Melanin reverses light in torsion fields}

Photon converts in the neutrino Melanin converts the light in the torsion field. Light travels in a vacuum, 300 thousand $\mathrm{km} \mathrm{sec}$ and the torsion field at 10 to $\mathrm{N}$, where $\mathrm{N}$ is the speed of light [36]. Torsion fields are conditioned by spin motion like the mass and spin charge is integrally linked to the particle. Spin is understood as own angular momentum of particles in the system. Each type of particle has an appropriate spin for itself. Spin is a purely quantum concept. In classical mechanics, the particle has zero angular momentum [37]. There are left and right torsion fields (depending on the direction of rotation of the spin). Molecular, atomic and nuclear spins determine intensity of the torsion field. According to Shipov every substance has its own characteristic of the torsion field. The torsion fields produce solitons. Transmission of soliton signal takes place not only to biological structures, but also to the psychological and spiritual realm- these are our mental, emotional and conscious states.

The soliton waves exhibit incredible resistance to distortion and noise interference. Solitons keep their shape and velocity after a collision with one other. They transmit signals without necessity to move the water environment as a carrier wave. Only the spatial relations, i.e. only geometry of the constellation of water molecules and air without their physical participation, are carriedenvironment participates "spiritually" as a structural pattern. Soliton waves have other properties - when two soliton waves approach one another, they "note" and penetrate themselves, but do not overlap; then they diverge in the same order in which they 
last. They penetrate just temporarily, without losing their identity Lomdahl 1984; Brizhik [38].

Solitons can propagate in the entire universe, without decay; they exist from the beginning of life up to the present. Space is densely filled with a network of solitons, carrying the content and meaning. Brain, and each replication of the genetic code system have transmitter and receiver antennas, which transmit space "directives" Edmundson, Enns, 1995. Signal transmission does not have to be made solely under the influence of electro-magnetic and acoustic wave or the electric field - in such a case solitons waves would function as an information carrier and could have an impact on the energy-informational system of a human being and its behavior $[9,39,40]$.

Human biological system is an integrated part of the piezoelectric and pyroelectric elements and semiconductors protein, nucleic acids, DNA, RNA, and melanins. In this circuit there is a biological system in the brain, central nervous system, which can control and coordinate the whole [21]. Control is accomplished by a grid of the following information channels: electron, photon, phonon, soliton, and free radical, and also to bioplasm; any channels of These may be the carrier of information for the biological system in itself, or function as a team in the bioplasm system Interpreted as a set of elementary particles and Mutually interacting fields [23]. Melanins and neuromelanins contain large density of bioplasm and this fact affects the development and degradation of human mental processes. Further understanding of melanins' nature will bring essential contribution to the development of psychology, especially in understanding which factors constitute the essence of the nature of the mental processes. Possession of melanins, neuromelanins and bioplasm in the body forces the science to the new interpretation of the functioning of the sensory perception mechanism and the nature of human mental processes. In summary, it is clear that the human psyche develops under the influence of energy factors and information of environment in which a person lives. The biological system plays a role of electronic devices and functions on an electronic material- piezoelectric, pyroelectric paramagnetic and semi conducting. Having this feature, it can transmit information to the inside and outside system by electronic means. A major function for the personal development is played by bioplasm:

A. Bioplasm has the capacity to adopt environmental information and to process it at its own information system. The transfer of this information can be realized by means of fields and quanta [23].

B. Bioplasm is the carrier of magnetic, electrical, chemical, mechanical, solitonic, optical, acoustic, gravity information as well as torsion fields Sedlak [41], Shipov 2004. Consciousness is a "headquarter" of bioplasm and a "bridge" between mind and brain. The nature of consciousness cannot be explained by classical concepts of psychology, since it is located in quantum processes. This fact forces psychology to take into account quantum physics, in order to better understand the human mental processes Figure 7.

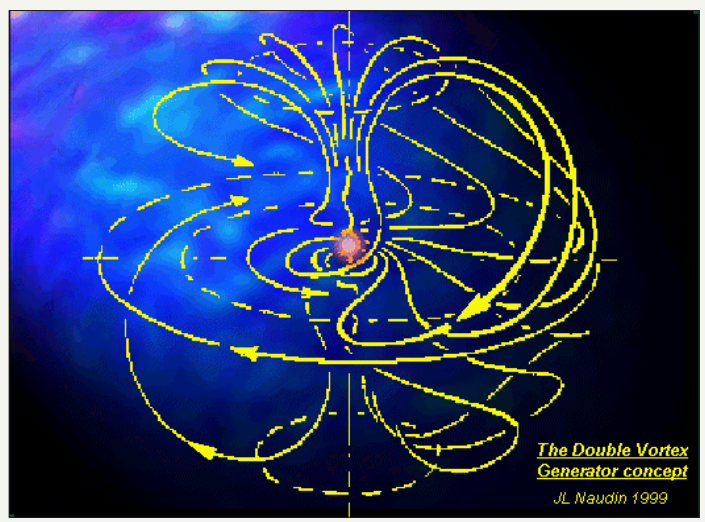

Figure 7: Shows the function bio plasm, which task is to coordinate, integrate, store and manage energy-informational processes in the human biological system.

\section{Syamala Hari 2008 - Tachyon concept of consciousness}

Although experiments to detect faster-than-light particles have not been successful so far, recently, there has been renewed interest in tachyon theories in various branches of physics. Hari 2008 suggests that tachyon theories may be applicable to brain physics. Eccles proposed that there is a link between psychons and dendrons which are basic anatomical units of the neocortex for reception. Hari proposes that a zero-energy tachyon could act as a trigger for exocytosis, not merely at a single presynaptic terminal but at all selected terminals in the interacting dendron by momentarily transferring momentum to vesicles, thereby decreasing the effective potential barrier and increasing the probability of exocytosis at all buttons at the same time. This is consistent with the view of tachyons, which treats them as strictly non-local phenomenon produced and absorbed instantaneously and non-locally by detectors acting in a coherent and cooperative way.

\section{Quantum models of consciousness [14]}

Vannini in her paper presents the characteristics of the quantum theory of consciousness and divides them into three categories:

1. Models which assume that consciousness creates reality and is dependent on sensory information and memory.

2. Models which link consciousness to the probabilistic properties of quantum mechanics;

3. Models which attribute essential entangled quantum state to consciousness

In her scientific output she demonstrated that consciousness creates the structure of quantum processes and links them with other mental processes.

\section{Marco Bischof-2008. Biofield concept of consciousness and its role in personal and collective interactions}

Bischof first proposed in his book "Biophotonen" (1995) that in addition to the electromagnetic biofield (biophoton field) there were one or several non-electromagnetic (or 'subtle') fields of psychophysical nature forming an interface between the 
physical body (solid and electromagnetic) and consciousness. He suggested scalar fields and electromagnetic potentials as possible candidates and described various concepts of the physical vacuum as a kind of global information field at the base of the physical universe. He also pointed out that several quantum features of the bioelectromagnetic field (biophoton field), particularly coherence, showed consciousness-like properties and could be considered as a "bridge to consciousness".

According to Bischof (1995) living systems may control their own space-time organization and life processes through a primary biofield of gravitation-like nature via the information fields of the non-electromagnetic biofields and the electromagnetic biofields. In 1998 he elaborated this concept and introduced the concept of "vacuum biophysics"-a new field of biophysics that should be "concerned with the investigation of the biological function of the physical vacuum and of the mediating role potentials and other nonelectromagnetic fields between the vacuum on the one hand and force fields and solid matter on the other hand" Bischof 1998. He suggested that the enfolded information structure of these quantum fields may control the electromagnetic biofield and the space-time organization and the physiological processes of the solid body. The origin of the measured electromagnetic biofields (biophotons) may be a quantum potential field or structured complex of vacuum states which ultimately is controlled by consciousness. In 2002 and 2003 Bischof presented a "Multidimensional Model of the Organism" based on these suggestions Bisch of 2002; Bisch of [15]. It postulates a hierarchy of levels of biological function, or regulation systems, in humans, and introduces levels of "subjective anatomy" (as in phenomenological thinking) in addition to the measurable levels of objective anatomy known to science.

Starting with the physical body, consisting of the solid-physical body of tissues, the humoral system of the liquid-colloid organism, and the bio plasmatic body of electric matter in the body, the next level is the electromagnetic body of the bioenergetic fields, followed by the non-electromagnetic body of bioinformation fields Besides these levels of "objective anatomy", the model additionally comprises a subjective anatomy part, consisting of subjective or "experienced bodies" roughly corresponding to the "subtle bodies" of Eastern medicine and religion, and the "self" (consciousness) as the deepest core of the person and highest regulatory instance. As first proposed by William James in 1890, Bischof conceives of consciousness as a field, but not necessarily of electromagnetic nature. He points out that this view is now accepted by many authors, some of which suggest it may possess the property of quantum non-locality. This model is not only based on the inclusion of consciousness, the intermediate levels of the bioenergetical and the bio informational fields, and the subjective aspects of our existence into the physical model of the organism, but also on a reversal of the usual primacy of matter over consciousness. [42] it proposes that consciousness may not be a (secondary) product of (primary) material brain structures, but rather an independent entity that uses the brain as an instrument to interact with the body and the material world. Bischof also further elaborated on the concept of vacuum biophysics. The vacuum is equated with the realm of "potentiality" beyond space-time which the Schrodinger wave equation describes and which according to [43] is the basis of the "actuality" of the observable world. This non-observable, unmanifested, pre-physical world of nonlocal correlations and general interconnectedness is the basis of the physical world and of matter, the fundamental dimension of reality [44].

It contains in potential form all the possibilities of physical manifestation. Bischof postulates that it is the foremost task of the new biophysics to arrive at an understanding of the process of manifestation from the realm of potentiality of the vacuum to the actuality of physical reality via the intermediate realm of the preparative phases of manifestation where this transposition happens, and which contains the intermediate pre-physical forms of manifestation. For many authors the vacuum seems also to be connected to consciousness. For this reason, some of them see the hidden domain of potentiality itself as a fundamental field of universal consciousness underlying physical reality, while others consider consciousness as an even more fundamental, separate reality behind the vacuum.

In two extensive papers in 2006 and 2008 Bischof claims that there is enough indirect and some direct evidence for the hypothesis that electromagnetic and non-electromagnetic biofields play also a crucial role in interpersonal relations and the social life of whole societies and postulates the necessity of developing a field theory of social life $[45,46]$. In his view, all human beings are at the unconscious level connected by a single consciousness field, individual consciousness existing only at the level of conscious awareness. We are much more open to influences operating at the unconscious level than we are aware of, unless we train our awareness of these influences, make them conscious, and learn to consciously respond to them or resist them.

\section{Hypothesis of virtual biophotons as the base for expla- nation of the nature of consciousness [46]}

The "Hard problem" of consciousness relates to the perplexing emergence of consciousness as a result of the brain activity. The binding problem concerns separate processes of perceived data in functionally and topographically segregated cortical areas and functional integration of such locally disjoined operations into coherently perceived images and events. The existing fieldgrounded theories of consciousness fall into two groups: (i) those based on the physical (electromagnetic) field, and (ii) those grounded on autonomous fields irreducible to the established physical fundamentals. The critical analysis of the existing theories results in formulation of the extra ingredient and a novel irreducible field-based theory of consciousness. Michael Lipkind proposes the hypothesis which says that virtual photons are responsible for the phenomenon of consciousness.

\section{Cybernetic-informative picture of consciousness [47]}

At the end of that monographs the role of awareness in the development of quantum artificial intelligence, biocybernetics, neural networks and biocommunication industry should also be mentioned. The development of these disciplines was in 
large measure possible thanks to prof. Richard Tadeusiewicz, who is a recognized authority and widely respected specialist in biocybernetics, automation and robotics and computer science. His interests are expressed in an enormous contribution to the writing of publications in various fields of modern science. Scientific achievements of Richard Tadeusiewicz is impressive and varied. His publication list includes over 800 works published in reputed national and international scientific journals and in the materials of the world congresses and conferences. There are more than 70 monographs, books, valued textbooks, but also translated books [48-60].

His publications and books on biocybernetics and biomedical engineering are now the source of inspiration for research in such fields as the identification and speech signal processing, and diagnostics based on analysis of sound signals. Tadeusiewicz is a leader of research in the field of neural networks since 1970s artificial intelligence, learning processes and their applications in medicine and biology. As far as bioinformatics is concerned, he suggested a new approach of automatic understanding of images that take into account the psychology of visual perception. It uses the fact that the human visual system in a more efficient process and interpret the information you previously acquired perceptual experience [61-90].

This replaces the automatic recognition of images by automatic understanding of images, this approach is original and unique in the world. Tadeusiewicz proves that in the image analysis systems, amount of information equals tens of millions of bits per second which can be compared with memory of a large computer. This information is recorded by retina and it is immediately subjected to treatment and reduction. It turns out that from those many millions bits of information only a dozen per second reaches the decisionmaking zone. Processing of information occurs beyond the level of our consciousness. It can be stated that some kind of automatism works there, which can be reconstructed in artificial control systems. Original results obtained in the field of digital imaging and its applications in machine vision systems, industrial robots. Gained global recognition methodologies automatic understanding of images in a monograph published by the prestigious publishing house Springer-Verlag 2004 [91-110].

According to Tadeusiewicz, very complex regulating, cybernetic and information processes occur on the level of a single cell. The processes of internal respiration, metabolism, metabolic control can be described in terms of cybernetics, the same information processing in processors, which are the main structure of the computer. These processes need the following elements to be involved in them: single molecules, protein structural that is a carrier of information, DNA and RNA acids and melanin. In medicine, a human being is usually viewed through the prism of the nineteenth-century biochemistry [111-130]. Our medicine is often a pill, the methods of acquiring information is mainly chemical analytics.

The body is treated as an object, in which one can observe the chemical processes neglecting the computer processes occurring in it. The better understanding of quantum consciousness can have a major impact on improving the work system of robots and machines, which are targeted by artificial intelligence. Together with cybernetics, it could be used in medicine - in the knowledge of metabolic processes and in processing, storing and managing of information occurring in psycho and biological processes [131150]. The processing of this information may be effected by artificial intelligence built in the structure of biological cells. According to the authors of the monograph closer understanding of the nature of consciousness allows to better understand the functioning of the human mental processes and also sensory perception.

It is concluded that in the process of seeing a very important role is played by melanin contained in the iris of the eye, but also in the epithelium of the retina. Iris of the eye and the retinal epithelium are composed of bio melanin, which is responsible for the management of information on the subconscious level, together with its diagnosis, the shaping and modification. Biocomputers melanin are powered by electricity generated in the metabolism and metabolic information in the vehicle for these nanocomputers an electromagnetic wave, solutions, and fields bioplasm, torsion Adamski 2009 [151-164].

\section{References}

1. Liberman EA (1996) Cell molecular computers and biological information as the foundation of nature's laws, Biosystems 38(2-3): 173-177.

2. Cardenas ML (1991) Are the transitory enzyme-complexes found invitro also transitory invivo. If so, are they physiologically important? Journal of Theoretical Biology 152(1): 111-113.

3. Athenstaedt H, Claussen H, Schaper D (1982) Epidermis of human skin: Pyroelectric and piezoelectric sensor layer. Science 216(4549): 10181020.

4. Athenstaedt H (1987) Spontaneous polarization and pyroelectric behavior of organisms, Ferroelectrics 73(1): 455-466.

5. Mc Guinnes JE, Corry P, Proctor P (1974) Amorphous semiconductor switching in melanins. Science 183(4127): 853-855.

6. Morowitz H (1978) Proton semiconductors and energy transduction in biological systems. American Journal of Physiology 235(3): 99-114.

7. Sedlak W (1976) Bioplasma. Materials from the $1^{\text {st }}$ conference dedicated to bioplasm. Catholic University of Lublin. Lublin, Poland.

8. Trąbka J (2001) Awareness of localizationism and globalism. Ed. Jagiellonian University, Poland, ISBN 83-233-1479-9.

9. Brizhik L (2002) Energy and information transfer in biological systems. How physics co enrich Italy: 18-22.

10. Mc Faden J (2002) The conscious electromagnetic information (Cemi) field theory. Journal of Consciousness Studies 9(8): 45-60.

11. Hu HP, Wu MX (2002) Spin-mediated consciousness Theory: possible roles of oxygen unpaired electronic spins and neural membrane nuclear spin ensemble in memory and consciousness. arXiv e-print.

12. Flanagan BJ (2003) Multi-scaling, quantum theory, and the foundations of perception. Neuro Quantology 1(4): 404-427.

13. Pereira A (2003) The quantum mind/classical brain problem. Neuro Quantology 1(1): 94-118.

14.Vannini A (2008) Quantum models of consciousness. Quantum Biosystems 2: 165-184. 
15. Bischof M (2003) Introduction to integrative biophysics. In: Popp FA, Belousova LV, (Eds.), Integrative biophysics. Kluwer Academic Publishers, Dordrecht, Netherlands, pp. 1-115.

16. Järvilehto $\mathrm{T}$ (2004) Consciousness and the ultimate essence of matter Neuro Quantology 2(3): 210-218.

17. Adamski A (2006) The role of bioelectronic processes in shaping sensual perception and mental functions of a human, University of Silesia Katowice, Poland.

18. Lauster P (1995) Self-awareness. Book World, Warsaw, Poland.

19. Zaborowski Z (1998) Awareness and self-awareness of man. Psychology and Culture, Warsaw, Poland.

20. Sedlak W (1968) Basics of the evolution of consciousness. Kosmos 17: 161-169.

21. Sedlak W (1980) Homo electronicus. Warszawa, Poland.

22. Sedlak W (1977) Piezoelectricity of organic compounds and quantum acoustic basics of biological information. Philosophical Annals 25(4): 149-170.

23. Sedlak W (1979) Bioelectronics 1967-1977. Warszawa, Poland.

24. Sedlak W (1973) The influence of consciousness on soma in bioelectronic context. Raised. Fiz and Sport 17(2): 69-77.

25. Inyushin WM (1974) Bioplasm and its contusion. In: Romen AC, (Ed.), Psichiczeskaja self-regulation. Alma-Ata 1974, Gosudarstwienny] University, Kazakh, p. 334.

26. Inyushin GU, Iliasow IA (1992) Niepomnjaszczich. Bioenergetics, structure, theory and practicality. Alma-Ata 1992, Gosudarstwienny) University, Kazakh, p. 110.

27. Inyushin WM (1978) Elements of the belyogyan field theory. Alma-Ata 1978, Goudroznnnyj University, Kazakh, p. 61.

28. Pilas B, Sarna T (1995) Quantitative determination of melanin in pigmented cells by electron spin resonance spectroscopy, In: Bagnara J Klaus SN, Paul E, Smart M, Giessen (Eds.), Proc 12, Int. Pigment Cell Conf, University of Tokyo Press, Tokyo, Japan, pp. 97-103.

29. Sarna $T$ (1992) Properties and function of the ocular melanin. A

30. King R (2001) Melanin a key freedom. Lushena Books, Chicago, USA.

31. Nicolas RA (1997) Colored organic semiconductors: Melanins. Rend. Academy of Physical and Mathematical Sciences. LXIV p. 342.

32. Strzelecka T (1982) Semiconductor properties of natural melanins Physiological Chemistry and Physics 14(3): 223-231.

33. Prota G (1993) Melanins and related metabolites in Black Skin. Pigment Cell Res 12: 73-99.

34. Schultz TM, Kurtz S, Wolfram LJ, Swartz H, Sarna T (1987) Paramagnetism in melanins: origin of the intrinsic free radical. First meeting of the European Society for Pigment Cell Research, Sorrento, October 11-14.

35. Prota G (2000) Melanins, melanogenesis and melanocytes. Looking at their functional Significance from the chemist's viewpoint. Pigment Cell Res 13(4): 283-293.

36. Shipov CI (1996) Unification of interactions in the theory of physical vacuum. Preprint Nr.3. MITPF.

37. Shipov GI (1995) Theoretical estimation of electrotorsion radiation. M: Preprint N1, MITPF. 21 p. 34-42

38. Brizhik L (1993) Soliton generation in molecular chains. Physical Review B 48(5): 3142-3144.

39. Adamski A (2005) Melanin, enzymes and melatonin in health and disease. Signum Magnum, Rybnik, Poland.

40. Brizhik L (2003) Soliton mechanism of charge, energy and information transfer in biosystem. World Scientific Publishing. Co Ptc Ltd, Singapore. Photobiophysical View. J Photochem Photobiol B 12(3): 215-258.

41. Sedlak W (1975) Bioplasma dynamics and metabolism. Kosmos A 24(3): 261-272.

42. Popper KR, Eccles JC (1977) The Self and Its Brain. New York, USA

43. Heisenberg W (1958) Physics and philosophy, Harper \& Row, New York, USA.

44. Friedman N (1997) The hidden domain. Woodbridge Group, Eugene, Oregon, USA.

45. Bischof M (2006) Vernadsky's noosphere and slavophile sobornost'some early concepts of field phenomena in social life. In: Beloussov LV, et al. (Eds.), Biophotonics and coherent systems in biology, New York, USA, pp. 279-297.

46. Bischof M (2008) Synchronization and coherence as an organizing principle in the organism, social interaction, and consciousness. Neuro Quantology 6(4): 440- 451.

47. Lipkind M (2008) Consciousness enigma: the hard problem--binding problem entanglement, extra ingredient and field principle. Indian journal of experimental biology 46(5): 395-402.

48. Tadeusiewicz R, Ogiela S (2006) Cognitive information systems for the analysis of CNS imaging, in the book, In: Grzech A (Ed.), Knowledge engineering and expert systems, Wroclaw University of Technology Publishing House, Wroclaw, Poland 1: 77-86.

49. Adamski A (2006) The biological system as an electronic device in learning about the environment and itself. Collective work under Adam Adamski. Ed. Propak Printing. Kety.

50. Adamski A (2006) Sensory and melatonin perception in the human biological system. Collective work under edited by Adam Adamski Edition Propak printing. Kety.

51. Kimov AE, Pougach AF (1992) The question of possibility of discovering of torsion waves by astronomic methods. Preprint CISE VENT, pp. 17-27.

52. Akimov AE (1995) Torsion communications of the third millennium. The proceedings of the international conference 'Modern Telecommunication Technologies, Moscow, Russia.

53. Bajpai RP (2003) Relevation of biophotons to consciousness; The physical basis of life; in integrative biophysics; Kluwer Academic Publishers, Netherland, pp. 439-465.

54. Bajpai R (2003) The physical basis of life and relevance of biophotons to concepts of consciousness, 2008 Indian Journal of Experimental Biology, 46, 420-432. Statistical Physics 29: 515-546.

55. Bielecki T (1988) The evolution of human brain - facts and speculations. Kosmos 3(3): 7-14

56. Bishop M (1995) Biophoton - the light in our cells. Two thousand, Frankfurt a Main.

57. Bishop M (1998) Scalar waves and quantum fields as a possible basis of biological information. Medical Association for Experience 47(6): 295300.

58. Bishop M (2002) Tachyon orgone energy Scalar waves-Subtle fields between myth and science. AT publishing house, Aarau, Switzerland, pp. 256-257.

59. Bischof M (2005) Biophotons-the light in our cells. Journal of Optometric Phototherapy.

60. Bischof M (2006) Interview conceded to Carlos augusto more of do not bother. Berlin, Germany.

61. Bohm D (1980) Wholeness and the implicate order, Routledge \& Kegan Paul London- Boston, USA.

62. Bondi M (1998) The role of synaptic junctions in the identification of human consciousness. Biology Forum 91(2): 329-334.

63. Bondi M (2005) Quantum electrodynamics and unified synaptic channel in the identification of consciousness, Neuro Quantology 3(2): 119-133.

How to cite this article: Adam Adamski. The Historical Aspect of the Development of Quantum Consciousness at the Beginning of the XXI Century. Gerontol \& Geriatric Stud .4(3). GGS.000588.2019. DOI:10.31031/GGS.2019.04.000588 
64. Bohr N (1965) Atomic physics and human knowledge Warsaw, Poland.

65. Buckley DH (1989) An electromagnetic theory of life. Medical Hypotheses 30(4): 281-285.

66. Buckley DH (1992) An electromagnetic theory of life-II: Testing. Medical Hypotheses 38(4): 305-310.

67. Caras S, Janata J (1988) Enzymatically sensitive field effect transistors. In: Mosbach K (Ed.), Immobilized enzymes and cells. San Diego Academic Press, California, USA 137: 247-255.

68. Conte Elio (2008) Testing quantum consciousness. Neuro Quantology 6(2): 126-139.

69. Cramer JG (1986) The transactional interpretation of quantum mechanics. Reviews of Modern Physics 58: 647-688.

70. Culbertson J (1963) The minds of robots. University of Illinois Press, USA.

71. Culbertson J (1976) Sensations, memories and the flow of time Cromwell Press, Towbridge, UK.

72. Fantappiè L (1991) Principles of a unitary theory of the physical and biological world. Di Renzo Editor, Rome, Italy.

73. Fantappiè L (1993) Selected conferences. In: Di Renzo (Ed.), Rome, Italy.

74. IFröhlich $H$ (1968) Long range coherence and energy storage in biological systems. Int J Quantum Chemistry 2(5): 641-649.

75. Fröhlich H (1988) Biological coherence and response to external stimuli. Springer, Berlin, Germany.

76. Gershon MD (1998) The Second Brain, Quill, New York, USA

77. Hameroff SR, Watt RC (1982) Information processing in microtubules Journal Theoret Biol 98(4): 549- 561.

78. Hameroff SR, Penrose R (1995) Orchestrated reduction of quantum coherence in brain microtubules: A model for consciousness. Neural Network. World 5(5): 793-804.

79. Hameroff S (1998) Quantum computation in brain microtubules: the Penrose- Hameroff model of consciousness. Phil Trans R Soc Lond 356(1743): 1869-1896.

80. Hameroff S, Penrose R (2003) Conscious events as orchestrated spacetime selections. Neuro Quantology 1(1): 10-35.

81. Hameroff S (2007) Orchestrated reduction of quantum coherence in brain microtubules. Neuro Quantology 5(1): 1-8.

82. Hameroff S, Chopra D, Penrose R (2010) The Universe and consciousness: quantum physics, evolution, brain \& mind.

83. Hu HP, Wu MX (2003) Spin-mediated consciousness theory: an approach based on panprotopsychism, Cogprints 4(1): 49-59.

84. Hu H, Wu M (2004) Spin as primordial self-referential process driving quantum mechanics, Spacetime Dynamics and Consciousness. Neuro Quantology 2(1): 41-49.

85. Huth (1984) Nonlinear tunneling barrier at high frequencies and their possible logic processing function in biological membranes. In: Adey R, Lawrence A (Eds.), Non-linear dynamics in biological systems, pp. 227 241.

86. Jacobson J (1989) On the electromagnetic nature of life. Panminerva Medicin 31(4): 151-165.

87. Jibu M, Yasue K (1995) Quantum brain dynamics and consciousness, in advances in consciousness. Research 3, John Benjamins Publishing Company, Amsterdam, Netherlands.

88. Jibu M, Hagan S, Hameroff SR, Pribram KH, Yasue K (1994) Quantum optical coherence in cytosceletal microtubules: implications for brain function. Biosystems 32(3): 195-209.

89. Kaivarainen A (1992) Mesoscopic theory of matter and its interaction with light. Principles of self-organization in ice, water and biosystems, University of Turku, Finland.

90. Kaivarainen A (2005) Hierarchic model of consciousness. Neuro Quantology 3(3): 180- 219.

91. King CC (1989) Dual-time supercausality. Physics Essays 2(2): 128-151.

92. King CC (1990) Did membrane electrochemistry precede translation? Origins of Life \& Evolution of the Biosphere 20(1): 15-25.

93. King CC (1991) Fractal and chaotic dynamics in the brain. Prog Neurobiol 36(4): 279-308.

94. King CC (1996) Fractal neurodyamics and quantum chaos: Resolving the mind brain paradox through novel biophysics, In: E Mac Cormac, Stamenov M (Eds.), Fractals of brain, fractals of mind, Advances in Consciousness Research 7, John Benjamin Publishing Company, Amsterdam, Netherlands.

95. King CC (1996) Quantum Mechanics, Chaos and the Conscious Brain. J Mind and Behavior 18(23): 155-170.

96. King CC (2003) Chaos, Quantum-transactions and consciousness. Neuro Quantology 1(1): 129-162.

97. Koch C, Hepp K (2006) Quantum mechanics in the brain, Nature 440(7084): 611.

98. Laszko E (1972) Introduction to systems philosophy. University Press, New York, USA.

99. Li K (1983) Indications of optical coherence in biological systems - In: Frohlich H, Kremer F (Eds.), Coherent excitations in biological systems, pp. 117-22.

100. Litt A (2006) Is the Brain a quantum computer? Cognitive Science 30(3): 593-603.

101. Lockwood M (1989) Mind, brain and the quantum. Basil Blackwell, Oxford, UK.

102. Marshall IN (1989) Consciousness and bose-Einstein condensates. New Ideas in Psychology 7(1): 73-85.

103. Marshall IN, Zohar D (1994) Quantum society. William Morrow, New York, USA.

104. Mc Fadden, Johnjoe (2000) Quantum evolution harpercollins. Final chapter on the quantum mind, ISBN 0-00-255948.

105. Mc Fadden, Johnjoe (2007) Journal: Neuro Quantology: 5(3): 262-270.

106. Mender D (2007) Decentering the subject of physics. NeuroQuantology 5(1): 175-181.

107. Molski M (2005) Non-traceability and biocherence. Philosophical Annals of the Catholic University of Lublin. Lublin T. LIII, Poland, pp. 207-212.

108. Moscow W, Ertel D, Adamski A (1998) Hypotheses on the essence of consciousness and bioelectronics. Bioelectronics Foundation Lublin, Poland.

109. Musumeci F, Brizhik L, Wan Ho (2002) Energy and information transfer in biological systems: How physics could enrich biological understanding: proceedings of the international workshop, Acireale, Catania, Italy, pp. 18-22.

110. Panov V, Kichigin V, Khaldeev G, Klyuev AV, Testov BV, et al. (1997) Torsion Fields and Experiments Journal of New Energy, Winter 2: 2939.

111. Penrose R (1989) The Emperor's new mind. Oxford University Press, United Kingdom.

112. Penrose R (2005) The great, the small and the human mind. Cortina Editore, Milano, Italy.

113. Penrose R, Hameroff S (2010) Consciousness in the Universe: neuroscience, Quantum Space-Time Geometry and Orch OR Theory. 
114. Pitkänen M (1990) Topological geometrodynamics. Internal Report, HU-TFTIR: 90-94.

115. Pitkänen M (2003) TGD (Topological geometry dynamics) Inspired Theory of Consciousness. Neuro Quantology 1(1): 68-93.

116. Poppel E (1989) Limits of consciousness. Warsaw, Poland.

117. Popp FA, Dürr HP, Schommers W (2002) What is Life? World Scientific, New Jersey-London-Singapore-Hongkong, China.

118. Popp FA, Chang JJ, Herzog A, Yan Z, Yan Y (2002) Evidence of nonclassical (squeezed) light in biological systems. Phys Lett A 293(1-2) 98-102.

119. Popp FA (2003) Food quality analysis using biophotonics. Food Technology: 7-8.

120. Popp FA, Belousova L (2003) Biophotonics. Kluwer Academic Publishers. Dordrecht, Boston, London.

121. Popp FA (2008) Consciousness as Evolutionary Process based on Coherent States. Neuro Quantology 6(4): 67-78.

122. Ricciardi LM, Umezawa H (1967) Brain and physics of many body problems. Kybernetik 4(2): 44-48.

123. Sarna THM, Swartz HM (1985) The Physical properties of melanins 25 333-357.

124. Schrödinger, Erwin (1959) Mind and Matter. Cambridge University Press, United Kingdom.

125. Sedlak W (1967) Model of the system emitting biological field and electrostasis. Kosmos 16: 151-159.

126. Sedlak W (1969) ABC of electromagnetic life theory. Kosmos A 18: 165-174.

127. Sedlak W (1971) Quantum bases of movement in the organic world. Philosophical Annals 19(3): 91-112.

128. Sedlak W (1972) Physical plasma as the basis for bioenergy. Annual. FILOZ 20(3): 125-148.

129. Sedlak W (1975) The evolution of bioplasma. Roczn. Philosopher 23(3): 95-116.

130. Sedlak W (1983) The nature of human consciousness in the light of bioelectronics. Philosophical Annals Philosophy of Nature 31(3): 8391.

131. Sedlak W (1988) Another way. Warsaw, Poland.

132. Sedlak W (1987) Lectures on bioelectronics, alias Almapress, Warsaw, Poland.

133. Sedlak W (2000) A small monograph on bioelectronics, published by Continuo, Radom

134. Sławinski J (1987) Electromagnetic radiation and the afterlife. Journal of Near-Death Studies 6(2): 127-133.

135. Sławinski J (1987) Response to commentaries on Electromagnetic radiation and the afterlife, J of Near-Death Studies 6(2): 127-133.

136. Sławinski J (1998) Electromagnetic nature of consciousness. In: Moskwa W, Ertel D, Adamski A (Eds.), Hypotheses on the Essence of Consciousness and Bioelectronics, Copyright 1998 by Foundation Bioelectronics im. W Sedlak, Lublin, Poland.

137. Sławiński J (1990) Photon circuit in biological systems, Bioelectronics: Materials of the $6^{\text {th }}$ Symposium, In: Sedlak W, Zon J, Wnuk M (Eds.) Catholic University of Lublin, Poland pp. 189-191.

138. Sławiński J (2001) Light and Life. Light and Optics in Biomedicine, In: Pluta M, Cysewska A (Eds.), Proceedings of SPIE No 4515: 1-7.

139. Sławinski J (2002) Endogenous ultraweak electromagnetic fields in biological systems; Bioenergetic and Information Aspectcts. In
Endogenous Physical Fields in Biology, International Sympozium Physical Fiuelds in Biology, Prague, pp. 47-49.

140. Sławinski J (2003) Biophotons from stressed and dying organisms: toxicological aspects. Indian Journal of Experimental Biology 41(5): 483-93.

141. Slawinski J (2006) Experiment overview, Hochschule Furtwagen, Germany.

142. Sławiński J, Górski Z (2008) Imaging of biophoton emission from electro stimulated skin acupuncture point jg4: effect of light enhancers. Indian J Exp Biol 46(5): 340-344

143. Shipov CI (1993) Theory of Physical Vacuum, M NT-Centr. Moskwa, Russia.

144. Shipov GI (1994) Proceedings of the sixth seminar Gravitational energy and gravitational waves. Doubna, Moskwa, Russia.

145. Tadeusiewicz R (2005) Is it possible to build an artificial brain. The Universe: a natural letter 106(1-3): 22-25.

146. Tadeusiewicz R (2006) Will artificial neural networks, being a model of the human intellect, bring us closer to the scientific understanding of the phenomenon of the soul? Chapter in the book In: Wójcik B (Ed.), Mind and Religion, Questions ad disputandum, No. 8, BIBLOS. Tarnów, 2006, pp. 15-29 and a voice in the discussion published on pp. 125-127 and 128-129.

147. Tadeusiewicz R (2004) Studying the functioning of brain with the help of neural networks, polish science joranda, college The Serena: 57-78.

148. Tadeusiewicz R, Rowiński T (2010) Psychology and computer science. Synergy and contradictions. [In Polish: Psychology and computer science. Synergy and contradictions]. Publisher of the Cardinal Stefan Wyszyński University, Warsaw, Poland.

149. Tadeusiewicz R (2009) Theoretical neurocybernetics [In Polish: Neurocybernetics theoretical], University Publisher Warsaw, Warsaw, Poland.

150. Tadeusiewicz R, Bolc L, Chmielewski LJ, Wojciechowski K (2010) Computer Vision and Graphics -Part 1, LNCS 6374, Springer-Verlag Berlin-Heidelberg- New York, USA.

151. Tadeusiewicz R, Rutkowski L, Scherer R, Zadeh LA, Zurda JM (2010) Artificial intelligence and soft computing, LNAI 6114, Springer-Verlag, Berlin-Heidelberg-New York, USA.

152. Tegmark M (2000) Importance of quantum decoherence in brain processes. Physical Review E 61: 4194-4206.

153. Trąbka J (1983) Mind and consciousness. Kracow, Poland.

154. Trąbka J (1991) The brain and his self. Jagiellonian University, Poland.

155. Trąbka J (2003) Light neuropsychology. Ed. Jagiellonian university. Kracow, Poland.

156. Van Wijk R (2002) The development of a bio-sensor for the state of consciousness in a human intentional healing ritual. Journal of the International Society of Life Information Science (ISLIS) 20(2): 694702

157. Van Wijk E (2004) Human biophoton emission. Recent Res. Devil. Photochem. Photobiol 7: 139-173.

158. Van Wijk R, Godeart G, Van Wijk EPA (2006) Human ultra-weak light emission in consciousness research. In: Locks J (Ed.), New Research on Consciousness. Hauppauge, NY: Nova Science Publishers: 233-269.

159. Van Wijk EP, Ackerman J, Van Wijk R (2005) Effect of meditation on ultraweak photon emission from hands and forehead. Frosch. Komplementarmed. Class. Naturehood 12(2): 107-112.

160. Vitello G (1995) Dissipation and memory capacity in the quantum brain model. International Journal of Modern Physics B 9(8): 973-989.

How to cite this article: Adam Adamski. The Historical Aspect of the Development of Quantum Consciousness at the Beginning of the XXI Century. Gerontol 
161. Vitello G (2001) My double unveiled-the dissipative quantum model of brain. Benjamins Publishing Co, Amsterdam. Netherlands.

162. Vitello G (2003) Quantum dissipation and information. A route to consciousness modelling. Neuro Quantology 1(2): 266-279.
163. Zon J (1986) Electron plasma in biological membranes. Ed. KUL Lublin, p. 76.

164. Zon J (2000) Bioplasms and physical plasma in living systems. Ed. R W KUL Lublin, p. 47. cc) (i) Creative Commons Attribution 4.0 International License

For possible submissions Click Here Submit Article

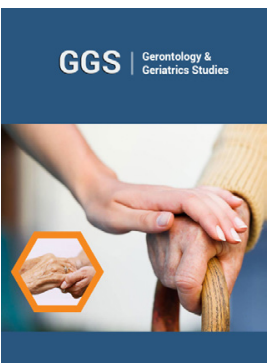

\section{Gerontology \& Geriatrics Studies}

Benefits of Publishing with us

- High-level peer review and editorial services

- Freely accessible online immediately upon publication

- Authors retain the copyright to their work

- Licensing it under a Creative Commons license

- Visibility through different online platforms 